\title{
Valoración del componente pragmático a partir de datos orales
}

\author{
B. Gallardo-Paúls
}

VALORACIÓN DEL COMPONENTE PRAGMÁTICO A PARTIR DE DATOS ORALES

\begin{abstract}
Resumen. Introducción. La evaluación pragmática suele resultar compleja, larga y bastante sofisticada, sobre todo para los profesionales sin formación lingüistica específica que se relacionan con los hablantes con déficit. Objetivo. Diseñar un método de evaluación rápido que permita una apreciación global rápida sobre la efectividad pragmática de los hablantes con afectación neurológica, de manera que este primer cribado permita decidir si es o no necesario un análisis en profundidad de las categorías alteradas. Desarrollo y conclusiones. Se parte de un perfil PerLA (percepción, lenguaje y afasia) de evaluación pragmática, diseñado para el análisis exhaustivo de datos conversacionales en lingüística clínica, y lo adaptamos a un modelo de cuestionario rápido. Se propone un modelo de valoración pragmática y se presentan los resultados obtenidos en un grupo de niños con trastorno por déficit de atención/hiperactividad. [REV NEUROL 2009; 48 (Supl 2): S57-61]

Palabras clave. Déficit lingüístico. Evaluación del lenguaje. Pragmática. TDAH.
\end{abstract}

\section{INTRODUCCIÓN Y OBJETIVOS}

En este trabajo analizamos las categorías lingüísticas que deben tenerse en cuenta para la valoración del lenguaje conversacional espontáneo en hablantes de edad infantil (incluyendo el lenguaje no verbal). Nuestro objetivo es describir los niveles comunicativos básicos en los que cabe identificar fallos pragmáticos desde una valoración inicial, a fin de incorporar la consideración de tales déficit en el diseño de la intervención logopédica. Asumimos que, en general, un déficit pragmático específico hace recomendable planificar una intervención más acorde con el paradigma comunicativo, mientras que si el déficit pragmático no es tan notable, o se debe claramente a limitaciones en el conocimiento gramatical, no lo consideramos un elemento central en la rehabilitación y, por el contrario, asumimos que es posible rentabilizar la pragmática para el éxito de la rehabilitación. Como veremos, la evaluación debería permitirnos separar los casos en que el déficit pragmático es específico, y los casos en que se asocia al déficit gramatical.

Nuestro punto de partida es el perfil PerLA de análisis pragmático $[1,2]$, que venimos utilizando para evaluar la competencia pragmática de los informantes del corpus PerLA ('percepción, lenguaje y afasia') en las categorías básicas de los tres niveles pragmáticos (enunciativo, textual e interactivo). Este perfil, no obstante, requiere un trabajo costoso y especializado, porque utiliza un nivel de análisis detallado que exige la cuantificación exacta de muchas categorías y que necesita siempre la transcripción de la interacción grabada; tales características explican que

\footnotetext{
Aceptado: 09.01.09.

Departamento de Lingüística General. Facultad de Filología, Traducción y Comunicación. Universitat de València. Valencia, España.

Correspondencia: Dra. Beatriz Gallardo-Paúls. Departamento de Lingüística General. Facultad de Filología, Traducción y Comunicación. Universitat de València. Avda. Blasco Ibáñez, 32, 5. ${ }^{\circ}$ E-46010 Valencia. Fax: +34 963864 778. E-mail: beatriz.gallardo@uv.es

Trabajo realizado en el marco de los proyectos de investigación financiados 'Coherencia, cohesión y pragmática textual en situaciones de déficit lingüístico'(MICINN, FFI2008-02592/FILO) y 'Protocolo de análisis pragmasintáctico. Un estudio de corpus oral' (MEC, ref. HUM2007-66074-C02-02).

(C) 2009, REVISTA DE NEUROLOGÍA
}

su aplicación sólo resulte posible en el ámbito de la investigación en lingüística clínica, pero no en la práctica rehabilitadora diaria.

Con la intención de simplificar este perfil PerLA, señalaremos algunas categorías básicas que sean susceptibles de una valoración rápida y no experta. Tal y como ocurre con otras pruebas pragmáticas, el diseño inicial para población adulta no excluye su aplicación a edades infantiles (por ejemplo, el protocolo pragmático de Prutting y Kirchner se diseñó para niños, pero se aplica actualmente a todo tipo de poblaciones). La idea básica que nos guía es proporcionar un instrumento útil, que suponga un primer paso de aproximación a la competencia comunicativa del paciente.

\section{DOS DÉFICIT PRAGMÁTICOS}

Nuestro planteamiento teórico es pragmático en sentido amplio, puesto que proponemos una consideración de categorías pragmáticas claramente dependientes de la habilidad gramatical. Dada la estrecha asociación entre todos los niveles del lenguaje, no resulta fácil aislar el déficit estrictamente pragmático. Con frecuencia, los déficit pragmáticos enunciativos o textuales pueden explicarse por limitaciones de naturaleza gramatical, por ejemplo:

- Alteraciones fonológicas: pueden impedir construir adecuadamente actos de habla proposicionales, cuyas unidades léxicas resulten inteligibles, subordinando la expresión a actos locutivos que se apoyan en interjecciones, entonaciones marcadas y vocalizaciones no léxicas.

- Alteraciones morfológicas: tradicionalmente asociadas al agramatismo, encuentran su dimensión pragmática provocando fallos en la cohesión lexicosemántica del discurso.

- Alteraciones sintácticas (paragramatismo, infradeterminación sintáctica): explican un déficit pragmático en la cohesión sintáctica.

- Fallos semánticos y de acceso léxico (hablantes con anomia o pobreza léxica): pueden explicar déficit en la construcción de los actos de habla proposicionales o de las estructuras sintácticas cohesionadas.

A su vez, estas alteraciones de carácter gramatical tienen también repercusión en otras categorías de naturaleza interactiva, como pueden ser la agilidad del turno (proporción de turnos por 
minuto en una conversación) o la distribución cuantitativa de los turnos de palabra (índice de participación conversacional). Así pues, la evaluación maneja rasgos que podremos caracterizar como de 'habilidad pragmática general' y otros que pueden describirse claramente como de 'habilidad pragmática de base gramatical'. Para cada paciente, la identificación de problemas en los respectivos ítems deberá conducir a evaluaciones detenidas mediante test específicos de cada componente.

Para los profesionales no familiarizados con la pragmática, cabe señalar que incluso en los casos de medición numérica de unidades y categorías pragmáticas -como en el perfil PerLA o la cuantificación de conductas conversacionales-, la evaluación pragmática depende de cómo el evaluador interpreta la adecuación texto/contexto, lo que, a su vez, debe utilizar como referente de 'normalidad' su propia conciencia de conversador habitual.

\section{NIVELES DE VALORACIÓN}

La primera sistematización que proponemos pasa por la diferenciación de tres niveles pragmáticos que vamos a valorar en el hablante. La situación óptima será aquélla en la que participen tres o cuatro interlocutores, y se adopte una estructura dialógica lo más parecida posible a una conversación. Los tres grandes bloques de la pragmática son los siguientes:

- Pragmática interactiva: son categorías pragmáticas que surgen al considerar que todo mensaje se dirige a un receptor, por lo que se centran en la toma de turno: agilidad del turno, índice de participación conversacional, prioridad o ajuste sociolingüístico, etc.

- Pragmática textual: son categorías específicamente vinculadas a la naturaleza gramatical del mensaje que construye el hablante, y que para tener tal valor de mensaje debe tener cohesión (adecuación léxica y cadenas correferenciales, estructuras morfosintácticas, conectores) y coherencia (superestructuras textuales).

- Pragmática enunciativa: son categorías que surgen por la consideración de cada enunciado como una acción intencional por parte de un hablante, es decir, los actos de habla y los significados inferenciales.

A continuación, presentamos las categorías de estos tres niveles de manera que puedan ser convertidas en un listado cumplimentado por el profesional que evalúa al niño; puede ser conveniente realizar las primeras valoraciones a partir de breves grabaciones, pero pronto resulta posible en presencia (por ejemplo, mientras se realiza la anamnesis inicial, o durante una sesión de consulta padres-niño-neuropediatra, el logopeda puede estar presente y realizar la evaluación).

\section{Pragmática interactiva}

En este nivel valoramos seis rasgos que nos informan sobre cómo el niño 'se engancha' en la toma de turno conversacional: agilidad del turno, índice de participación conversacional, predictibilidad, gestualidad, mirada y adecuación sociolingüística. Los estudios sobre adquisición [3,4] señalan que desde los 8-9 meses es posible identificar una toma de turno preverbal, y que ésta se puede estabilizar entre los 2:6 y los 3:6 años.

\section{Fluidez o agilidad del turno}

Se mide exactamente como número de turnos por minuto, y se contrasta con las medidas obtenidas en la conversación cotidia- na normal; obviamente, la medida exacta necesita la transcripción, por lo que en esta valoración nos limitamos a señalar si, a nuestro juicio, la situación concreta del paciente provoca un considerable enlentecimiento respecto de la habitual toma de turno conversacional. Es necesario tener en cuenta que, en la conversación con un adulto, el bebé es capaz de contribuir al desarrollo conversacional (mantener y seguir el tema) a partir de los 2 años. Sin embargo, hasta la edad de 5 años son normales los lapsos, es decir, rupturas de la toma de turno que provocan espacios de silencio conversacional; también desde esta edad podremos esperar que los niños y niñas rectifiquen los posibles fallos en la alternancia de turno.

\section{Índice de participación conversacional}

Valoramos aquí el nivel de participación en el intercambio conversacional y el interés por la comunicación. Se marca negativamente en dos situaciones típicas:

- Cuando es evidente que el niño participa muy poco en la conversación, construyendo turnos bastante breves que se limitan a dar respuestas mínimas a lo que se le pregunta, o emisiones breves que demuestran su escucha comprensiva ('claro, ya, ajá, sî'), es decir, cuando la impresión general sea de una toma de turno muy descompensada, de baja participación.

- Cuando la participación conversacional sea claramente excesiva, monopolizando el turno y dificultando la participación de los demás (logorrea).

\section{Predictibilidad}

Con este rasgo reflejamos si el habla del niño muestra un relativo equilibrio entre intervenciones de respuesta (turnos reactivos) e intervenciones de propia iniciativa (turnos iniciativos: preguntas o informes). Se valora negativamente tanto en los casos en que el niño se limita a responder, sin demostrar interés en ceder el turno al interlocutor, como en los casos en que la logorrea o la impulsividad obstaculizan gravemente la participación de los demás; también en situaciones en que el hablante se expresa sin demostrar vínculos entre lo que dice y lo que han dicho antes sus interlocutores. Hemos de tener en cuenta aquí variables como la situación (sería esperable que en el contexto de la consulta el niño pueda estar más inhibido que en situaciones familiares) o los interlocutores (si son todos adultos o el niño habla con otros niños).

\section{Gestualidad}

Este ítem se refiere al uso de los códigos visuales y gestuales (movimientos, expresividad facial); se valora positivamente en casos en que el lenguaje se apoya en los gestos de manera natural, pero negativamente cuando se aprecia bien un uso enfático, desmesurado, de estos mecanismos (por ejemplo, por hiperactividad), o bien un uso reducido y descompensado (por ejemplo, por hemiplejía corporal, apatía o distonía).

\section{Mirada}

En este apartado consideramos aisladamente el uso de la mirada como elemento comunicativo, que informa sobre la escucha activa del hablante, su solicitud de participar en la conversación, su petición y cesión del turno...

\section{Prioridad y adecuación sociolingüística}

A partir de los 2 años, el niño empieza a saber adaptarse al estilo de habla de su interlocutor y a las necesidades del contexto, y 
a utilizar formas corteses (con variaciones), que se estabilizan en torno a los 9 años. Se indicará negativamente en los casos en que la conducta verbal se aleje claramente de las normas sociales: indiscreciones, groserías, descortesía, 'salidas de tono', etc.

\section{Pragmática textual}

En este punto valoramos el alcance pragmático de las categorías y unidades gramaticales, por lo que debemos tener siempre muy presente la edad del niño y la adquisición de la lengua en el desarrollo típico. Analizamos cuestiones de morfosintaxis, semántica, informatividad, cohesión y coherencia.

\section{Cohesión morfológica: agramatismos}

Este ítem se refiere a la estructura morfológica de las palabras, lo que en lenguas como el español, catalán o gallego se plasma en la utilización adecuada de los morfemas o desinencias gramaticales, que aportan la información de género (femenino o masculino), número (singular o plural), tiempo verbal (perfecto, imperfecto, presente, futuro...), etc. Obviamente, la valoramos negativamente en los casos en que el niño muestra clara vacilación en la selección de morfos gramaticales o dificultades selectivas para ciertas categorías morfológicas que no pueden explicarse por el estadio de desarrollo en que se encuentra.

\section{Cohesión sintáctica: paragramatismos}

Este rasgo se relaciona con la organización sintáctica ('horizontal') del enunciado, es decir, la construcción de turnos conversacionales que se ajustan a la sintaxis de la gramática; es importante señalar aquí, no obstante, que nos referimos a una sintaxis conversacional, bastante menos rígida que la del texto escrito. Por lo tanto, sólo valoraremos este apartado como deficitario en los casos en que la organización sintáctica resulta claramente desestructurada, impidiendo la correcta decodificación, con oraciones frecuentemente inconclusas y abundancia de repeticiones y reinicios.

\section{Cohesión lexicosemántica}

En este ítem calibramos si el niño tiene la disponibilidad léxica propia de su edad; por tanto, la marcamos negativamente en todos los casos de anomia, fallos léxicos, parafasias semánticas, etc., que afecten claramente a la fluidez y que no puedan explicarse por la edad. También se valora negativamente cuando se aprecia un alto predominio de expresiones rutinarias, muletillas, palabras sin contenido... que rellenan el turno, pero en realidad no aportan información. Los estudios longitudinales indican que la referencia anafórica presenta vacilaciones hasta al menos los 6 años, y los conectores o marcadores discursivos se afianzan a partir de los 6-7 años; con todo, son esperables errores de cohesión referencial hasta los 9-12 años.

\section{Coherencia textual: gestión temática}

La gestión temática se relaciona con la habilidad del niño para desarrollar un tema, terminarlo, proponer uno nuevo, etc. Valoramos negativamente, por ejemplo, los casos en que el evaluado no sabe desarrollar los temas conversacionales que surgen (decimos que no los 'topicaliza') y sus turnos parecen no guardar relación con los turnos de los demás; también cuando la actitud comunicativa general transmite desinterés y no se aportan informaciones nuevas. Nuevamente es necesario tener en cuenta, como referencia, la habilidad mostrada por los niños de cada edad, sin utilizar el habla adulta como modelo [5]; como hemos dicho, los estudios de adquisición indican que desde los 2 años el niño ya sabe realizar estas topicalizaciones, aunque sea con una gramática deficiente, pero hasta los 9 años no se consolida la capacidad para identificar la relevancia informativa de cada idea.

\section{Coherencia textual: superestructuras}

Las superestructuras son esquemas mentales de naturaleza textual que nos indican las partes básicas de cada tipo de texto; por ejemplo, una narración exige como mínimo una complicación y una resolución, unos personajes, un principio y un final... [6-8]; en una argumentación utilizamos premisas o justificaciones y conclusiones, puntos de partida, ejemplos, refuerzos... Estas categorías pueden aparecer en un orden no lógico, incoherente, o pueden estar incompletas. Para su evaluación, planteamos al niño preguntas desencadenantes típicas del tipo ‘QQué has hecho este fin de semana?', o, en el ámbito argumentativo, ‘¿por qué has dicho que...?'. En casos de gravedad no es posible evaluar esta categoría, porque no hay suficiente fluidez como para que el hablante encadene las oraciones mínimas. Valoramos negativamente el discurso desorganizado, sin aparente estructura o con estructura incompleta; desde los 5-7 años, el niño es capaz de construir textos simples, acordes con la superestructura narrativa, pero la argumentativa se instaura algo más tarde (aquí puede haber cierta determinación cultural, ya que vivimos en un contexto sociocultural más narrativo que argumentativo [9]).

\section{Pragmática enunciativa}

Comenzamos la valoración del nivel enunciativo con los actos de habla, que son la unidad básica. La bibliografía sobre adquisición indica que desde los 12 meses [10] el niño manifiesta ya cierta intencionalidad comunicativa, y hasta los 32 meses se produce el desarrollo de los tipos básicos de actos de habla (declaraciones, interrogaciones o peticiones), cuyo inventario puede considerarse consolidado entre los 3 y 4 años. Desde los 2 años, el niño empieza a utilizar actos de habla de aclaración-justificación, y ya es capaz de responder a peticiones de información no específicas.

\section{Actos de habla locutivos enunciativos}

Consideramos, en primer lugar, la existencia de problemas motores que puedan afectar a los órganos bucofonatorios que participan en la articulación verbal, o también problemas de modulación, ritmo y control de la voz (entonaciones extrañas o descompensadas, disfluencias); marcamos negativamente los problemas que impiden construir normalmente los actos de habla locutivos enunciativos, es decir, encargados de la mera articulación oral, sonora.

\section{Actos de habla locutivos proposicionales}

Reflejamos aquí los problemas de anomia que dificulten el acceso léxico. Si no existen tales problemas, el niño construirá los actos proposicionales con una selección léxica propia de su edad.

\section{Limite entre actos de habla: uso de pausas}

En este apartado valoramos un uso anómalo o marcado de pausas, por ejemplo, cuando observamos un habla entrecortada salpicada de silencios que rompen el desarrollo temático, sin que esta discontinuidad sea típica de la edad (desde los 5 años, aproximadamente).

\section{Actos no verbales}

Antes hemos valorado el uso de la gestualidad; en este apartado analizamos una utilización de lo no verbal que surge específica- 
mente como compensación de la realización deficitaria de los actos verbales. El niño recurre a los gestos utilizando más de lo habitual emblemas (gestos fijos, que pueden sustituir a las palabras, con un significado de diccionario), reguladores (gestos que sirven para gestionar la toma de turno) e ilustradores (gestos que acompañan el habla y completan su sentido). Sin embargo, sólo cabe esta valoración en los casos en que hayamos valorado negativamente el uso de los actos de habla (por ejemplo, en el caso de la excesiva gesticulación y movilidad de niños hiperactivos, puede que no valoremos estos actos no verbales, pero sí hayamos valorado negativamente la gestualidad propia del primer bloque).

\section{Actos de habla ilocutivos}

Señalamos afirmativamente la utilización del lenguaje que refleja comprensión adecuada, y una expresión ajustada a la intención comunicativa; esa intención (llamada acto ilocutivo), obviamente, sólo la deducimos de la situación. Los estudios de adquisición señalan que esta intencionalidad es apreciable ya desde los actos preverbales de los 12-14 meses.

\section{Autorrectificaciones conversacionales}

Valoramos positivamente los casos en que el niño o niña muestra conciencia de su propia dificultad, iniciando él mismo rectificaciones en los puntos problemáticos y explicitando comentarios metalingüísticos que se justifican por los errores ('no, no es eso', 'ay, me he equivocado', 'no me sale', 'ya me he vuelto a equivocar', etc.). Incluimos este rasgo en las categorías enunciativas, porque los errores suponen una inadecuación entre lo dicho (el mensaje) y la intención comunicativa del sujeto, y las autorrectificaciones ponen de manifiesto que él es consciente del desajuste entre ambos. La conciencia metapragmática se puede esperar desde los 6-7 años.

\section{Inferencias trópicas o modismos}

Pertenecen al léxico de la lengua, y pueden verse afectadas en ciertas situaciones. Para evaluar estas categorías, el logopeda suele introducir en su discurso algún comentario que incluya el uso de estas expresiones idiomáticas que todo hablante suele conocer (por ejemplo, 'no dar el brazo a torcer', 'coger algo por los pelos', 'meter la pata', 'dar en la nariz', 'de cine'... expresiones de argot juvenil). No se trata de preguntar lo que significan estas expresiones, sino de usarlas en una intervención y comprobar que no plantean problemas de comprensión al paciente. Igualmente, si es él quien las utiliza en su conversación, lo valoramos afirmativamente. Obviamente, en el caso de la evaluación infantil hemos de tener en cuenta la adecuación de las expresiones a la edad del niño, ya que la correcta adquisición de estas expresiones se puede extender desde los 5-6 hasta los 17 años.

\section{Implicaturas conversacionales}

Este ítem hace una evaluación general sobre el respeto de las máximas conversacionales, dependientes del principio de cooperación formulado por Grice: 'Haz tu contribución conversacional tal y como es requerida, en el momento del intercambio en que te hallas implicado, y según la finalidad previamente aceptada de tal intercambio'. Las cuatro máximas se evalúan por separado:

- Máxima de la cualidad: relativa a la idea de que hablamos diciendo la verdad, se respeta previsiblemente por los hablantes sin daño cognitivo.

- Máxima de la cantidad: la marcamos negativamente tanto en los casos de logorrea (información excesiva o poco rele-
Tabla. Cuestionario de valoración pragmática.

\begin{tabular}{|c|c|c|}
\hline & Sí & No \\
\hline \multicolumn{3}{|l|}{ Pragmática interactiva } \\
\hline \multicolumn{3}{|l|}{ 1. Fluidez o agilidad del turno } \\
\hline \multicolumn{3}{|c|}{ 2. Índice de participación conversacional } \\
\hline \multicolumn{3}{|l|}{ 3. Predictibilidad } \\
\hline \multicolumn{3}{|l|}{ 4. Gestualidad } \\
\hline \multicolumn{3}{|l|}{ 5. Mirada } \\
\hline \multicolumn{3}{|c|}{ 6. Prioridad y adecuación sociolingüística } \\
\hline \multicolumn{3}{|l|}{ Pragmática textual } \\
\hline \multicolumn{3}{|c|}{ 7. Cohesión morfológica: agramatismos } \\
\hline \multicolumn{3}{|c|}{ 8. Cohesión sintáctica: paragramatismos } \\
\hline \multicolumn{3}{|l|}{ 9. Cohesión léxicosemántica } \\
\hline \multicolumn{3}{|c|}{ 10. Coherencia textual: gestión temática } \\
\hline \multicolumn{3}{|c|}{ 11. Coherencia textual: superestructuras } \\
\hline \multicolumn{3}{|l|}{ Pragmática enunciativa } \\
\hline \multicolumn{3}{|c|}{ 12. Actos de habla locutivos enunciativos } \\
\hline \multicolumn{3}{|c|}{ 13. Actos de habla locutivos proposicionales } \\
\hline \multicolumn{3}{|c|}{ 14. Límite entre actos de habla: uso de pausas } \\
\hline \multicolumn{3}{|l|}{ 15. Actos no verbales } \\
\hline \multicolumn{3}{|l|}{ 16. Actos de habla ilocutivos } \\
\hline \multicolumn{3}{|c|}{ 17. Autorrectificaciones conversacionales } \\
\hline \multicolumn{3}{|c|}{ 18. Inferencias trópicas o modismos } \\
\hline \multicolumn{3}{|l|}{ 19. Máxima de la cualidad } \\
\hline \multicolumn{3}{|l|}{ 20. Máxima de la cantidad } \\
\hline \multicolumn{3}{|l|}{ 21. Máxima de la manera } \\
\hline 22. Máxima de la pertinencia & & \\
\hline 23. Implicaturas anómalas & & \\
\hline
\end{tabular}

vante), como en casos de hablantes que apenas hablan con monosílabos y se limitan a turnos de respuesta, de manera que hay que insistir en las preguntas para que den la suficiente información (dan respuestas 'de mínimos').

- Máxima de la manera: se refiere, estrictamente, al uso de un estilo comunicativo claro y conciso, que evita la expresión farragosa o dispersa y las ambigüedades. En general, es una categoría de gran dependencia gramatical, por lo que su evaluación es con frecuencia negativa, por ejemplo, en las afasias o en ciertos hablantes con glosomanías y gran especialización léxica (síndrome de Williams, síndrome de Asperger).

- Máxima de la pertinencia: exige construir intervenciones relevantes para el tema en curso, de manera que la valoramos negativamente en los casos de rupturas temáticas bruscas, glosomanías o temas preferidos que obstaculizan el desarrollo fluido de los temas, etc. También se ve truncada, obviamente, en las situaciones donde falla notablemente la comprensión. 


\section{Implicaturas anómalas}

Las cuatro máximas anteriores se utilizan a veces a partir de su transgresión: son los sentidos figurados que improvisa cualquier hablante en su discurso, y que los niños de desarrollo típico adquieren entre los 4-6 años. Como en el caso de los modismos, el profesional puede optar aquí por la emisión de intervenciones que contengan este tipo de usos; por ejemplo, puede introducir expresiones que incluyan metáforas, hipérboles ('hace mil años que me dedico a esto'), ironías ('con lo poco/mucho que me gusta a mí...', 'con el calor/frío que hace hoy...', diciendo lo contrario de la realidad) o, en general, usos figurados. Hay situaciones deficitarias, como los trastornos de espectro autista, en que estos usos figurados se interpretan en sentido literal, por lo que es conveniente incluir su valoración.

\section{CUESTIONARIO}

Podemos concretar la valoración de estas categorías en un cuestionario que permita una valoración simple, en términos de afirmación/negación -algo habitual en los protocolos pragmáticos(Tabla). A partir de ahí, podemos establecer porcentajes, que nos permiten diferenciar tres medidas:

- Habilidad pragmática general: resultado de dividir las respuestas afirmativas entre los ítems evaluados y multiplicar por 100. La mayor habilidad corresponde a las medidas altas.

- Habilidad pragmática específica: reduce el inventario de categorías pragmáticas, eliminando aquéllas cuya ejecución necesita por completo el dominio léxico, semántico, fonológico y morfosintáctico. Son los ítems 1-6 (pragmática interactiva), 10-11 (pragmática textual) y 15-23 (pragmática enunciativa, salvo el 21), y que se miden con la misma operación referida para la habilidad general.

- Habilidad pragmática de base gramatical: engloba las categorías cuya eficacia depende de una habilidad previa en los componentes gramaticales; se realiza la misma operación para los ítems 7-9, 12-14 y 21 (pragmática textual y enunciativa).

\section{VALORACIÓN DE NIÑOS CON TRASTORNO POR DÉFICIT DE ATENCIÓN/HIPERACTIVIDAD}

Hemos valorado con este modelo 13 grabaciones de hablantes con diagnóstico de trastorno por déficit de atención/hiperactividad combinado, con una media de edad entre 9 y 11 años, y procedentes del Instituto Valenciano de Neurología Pediátrica y el centro CADAN de Badajoz. Los resultados confirman algunas de las apreciaciones repetidas por la bibliografía sobre el tema, referentes a un fallo más pragmático que gramatical en estos niños.

Utilizando el cuestionario que hemos descrito, encontramos que en las categorías interactivas, la impulsividad y la hiperactividad explican la gestualidad excesiva y las dificultades para el respeto de la toma de turno. En el ámbito textual, lo más notable es la transgresión en el uso de las superestructuras y, en ocasiones, el uso erróneo de los conectores, así como algunos fallos en la cohesión léxica correferencial. Por último, las categorías enunciativas son las que menos problemas presentan, ya que los niños de nuestra muestra no presentan problemas en la confección de actos de habla proposicionales ni en la ejecución de los actos ilocutivos; sin embargo, la máxima conversacional de la cantidad sí ofrece cierto nivel de desajuste, que tal vez sea explicable por la situación comunicativa que hemos recogido, donde el niño relata a su logopeda una historia que ha escrito (se aprecia cierta resistencia informativa, el logopeda se ve obligado a obtener la información utilizando versiones de insistencia).

La utilización de este cuestionario en una muestra representativa de informantes puede contribuir a establecer medidas de habilidad pragmática en el trastorno por déficit de atención/hiperactividad, discriminando las alteraciones estrictamente pragmáticas de las que tienen base gramatical, y repasando en detalle cada una de las categorías. Creemos, en definitiva, que este modelo permite una valoración rápida del componente pragmático, que es a la vez sistemática (organiza las categorías evaluadas en tres niveles con justificación teórica), exhaustiva (incluye todos los elementos importantes del componente) y fácil de aprender para profesionales sin formación específica en lingüística.

\title{
BIBLIOGRAFÍA
}

1. Gallardo-Paúls B. Más allá de las palabras y su estructura: las categorías del componente pragmático. In Garayzábal E, ed. Linguiística clínica y logopedia. Madrid: A. Machado Libros; 2006. p. 81-196.

2. Gallardo-Paúls B. Pragmática para logopedas. Cádiz: Publicaciones de la Universidad de Cádiz; 2007.

3. Adams C. Practitioner review: the assessment of language pragmatics. J Child Psychol Psychiatry 2002; 43: 973-87.

4. Serra M, Serrat E, Solé R, Bel A, Aparici M. La adquisición del lenguaje. Barcelona: Ariel; 2000.

5. Fernández-Pérez M. Dinamismo construccional en el lenguaje infantil y teoría lingüística. ELUA 2003; 17: 273-87.

6. Kaderavek JN, Sulzby E. Narrative production by children with and without specific language impairment: oral narratives and emergent readings. J Speech Hear Res 2000; 43: 34-49.
7. Merritt DD, Liles BZ. Story grammar ability in children with and without language disorder: story generation, story retelling, and story comprehension. J Speech Hear Res 1987; 30: 539-52.

8. Tannock R, Purvis K, Schachar R. Narrative abilities in children with attention deficit hyperactivity disorder and normal peers. J Abnorm Child Psychol 1993; 21: 103-17.

9. Gallardo-Paúls B. Huellas lingüísticas de teoría de la mente. Rev Neurol 2008; 46: 29-35.

10. Adams C, Bishop DV. Conversational characteristics of children with semantic-pragmatic disorder. 1: Exchange structure, turntaking, repairs and cohesion. Br J Disord Commun 1989; 24: 211-39.

\section{ASSESSMENT OF PRAGMATICS FROM VERBAL SPOKEN DATA}

\begin{abstract}
Summary. Introduction. Pragmatic assessment is usually complex, long and sophisticated, especially for professionals who lack specific linguistic education and interact with impaired speakers. Aim. To design a quick method of assessment that will provide a quick general evaluation of the pragmatic effectiveness of neurologically affected speakers. This first filter will allow us to decide whether a detailed analysis of the altered categories should follow. Development and conclusions. Our starting point was the PerLA (perception, language and aphasia) profile of pragmatic assessment designed for the comprehensive analysis of conversational data in clinical linguistics; this was then converted into a quick questionnaire. A quick protocol of pragmatic assessment is proposed and the results found in a group of children with attention deficit hyperactivity disorder are discussed. [REV NEUROL 2009; 48 (Supl 2): S57-61]
\end{abstract}

Key words. ADHD. Language evaluation. Linguistic deficit. Pragmatics. 